\title{
Molecular Recombination in T4 Bacteriophage Deoxyribonucleic Acid
}

\author{
I. Tertiary Structure of Early Replicative and Recombining \\ Deoxyribonucleic Acid
}

\author{
ANDRZEJ W. KOZINSKI, P. B. KOZINSKI, AND R. JAMES \\ Department of Medical Genetics, University of Pennsylvania, Philadelphia, Pennsylvania 19104
}

Received for publication 11 March 1967

\begin{abstract}
A replicative hybrid resulting from the infection of heavy (substituted with 5bromodeoxyuridine) bacteria with light (not substituted with 5-bromodeoxyuridine) radioactive bacteriophage was isolated from a $\mathrm{CsCl}$ density gradient. Sedimentation studies indicate that $60 \%$ of the deoxyribonucleic acid (DNA) behaves as if it were in units more than four times as large as an intact reference molecule. Under the electron microscope, hybrid molecules appeared tangled, showed puffs and loops, occupied a small area, and often had a total length twice that of mature phage. This indicates that sucrose gradient sedimentation is not applicable as a method for estimating the relative molecular size of replicative forms of DNA. After denaturation, the separated strands of hybrid were of the same size as those of reference DNA. $\mathrm{CsCl}$ density gradient analysis revealed no terminal covalent addition of new material to the old parental strand. The possibility of a continuous growth of the DNA molecule, either on a single-stranded level or as a double helical structure, is disproved. When chloramphenicol (CM) was added at critical times after infection, DNA synthesis continued at a constant rate. The parental label soon assumed and retained a hybrid density, despite concomitant synthesis of DNA, throughout the rest of the period of incubation in CM. The hybrid moiety, however, actively participated in replication and exchanged its partner strand for a new one; this was demonstrated by changing the density label during incubation in CM. A new enzyme synthesized shortly after infection introduced single-stranded "nicks" into the parental DNA. Since nicking can be inhibited by chloramphenicol, the responsible enzyme is not of host origin.The time of the appearance of this enzyme coincided with the onset of molecular recombination. Another enzyme, which mediates the repair of the continuity of the polynucleotide chain after recombination, appeared after recombination. If selectively inhibited by chloramphenicol, recombinant molecules remained unrepaired, and, upon denaturation, the parental fragment was liberated in pure form.
\end{abstract}

Frankel (5) and Thomas (16) postulated a mode of replication of $\mathrm{T} 4$ bacteriophage deoxyribonucleic acid (DNA) in which the parental molecule does not divide after duplication, but, as synthesis proceeds, continues to elongate into a molecule with a mass far exceeding that of normal phage. The appealing aspect of this hypothesis was the possibility of explaining the circular permutation of $\mathrm{T} 4$ molecules. If an endonuclease of undefined origin somehow introduced equally spaced double-stranded scissions into this very large polymer, mature phage molecules would result. The hypothesis of Frankel and Thomas was derived from the unusual sedimentation characteristics of replicative DNA in a sucrose gradient. When compared with ref- erence DNA, replicative DNA appeared to be of a very "large size," sedimenting at a rate more than that expected for 4 phage equivalent units.

Replication in this manner would provide a notable exception to any mechanism of replication described so far. We felt that a critical evaluation of the application of sucrose sedimentation, as a tool for measuring relative molecular sizes of intracellular DNA, should be performed on isolated replicative forms of DNA. For this purpose, the most useful replicative form would be hybrid, since its composition of one parental and one new strand has been well described $(8,9)$.

If the parental molecule divides into two prog- 
eny molecules immediately after duplication, the resulting hybrid molecule will be equivalent in size to one phage complement. If after one round of replication (see Materials and Methods) the parental strands somehow remain linked, a maximal size of two will occur. This last possibility will be considered more extensively in the Discussion.

An experiment in which molecules replicate continuously, in a medium in which progeny DNA differs in density from parental DNA, should provide a test for the hypothesis of Frankel and Thomas. If they are correct, the stage at which the parental label has a hybrid density should be only a transitory one. As the molecule increases in length, the hybrid section will become adjacent to areas of the molecule formed exclusively from new precursors. This would change the density of the molecule from hybrid to a density determined by the amount (length) of newly added DNA. The hypothesis of continuous elongation of the molecule does not provide any model for the exchange of partner strands in the hybrid segment. We therefore designed an experiment to test whether a stable form of hybrid can occur that will persist during the concomitant synthesis of DNA. We also investigated whether such a hybrid would or would not exchange its partner strand for a new one.

Because it is reasonable to suppose that the introduction of single-stranded breaks is a prerequisite for recombination, a related group of experiments was devoted to examining the appearance and repair of single-stranded nicks before and during molecular recombination. We feel that we should also present a thorough description of a phenomenon compatible with this theory, which we only casually commented on in our previous paper (13).

We have observed that there is a gradient of inhibitory activitites of chloramphenicol (CM). First, when $\mathrm{CM}$ is added between 0 and $5 \mathrm{~min}$ after infection, there is no replication of DNA. When it is added at $5 \mathrm{~min}$ after infection, replication occurs, but molecular exchanges within the vegetative pool are inhibited. When $\mathrm{CM}$ is added at approximately $7 \mathrm{~min}$ after infection, molecular exchanges occur, but the integrity of the polynucleotide chain is not restored. This is deduced from the fact that after denaturation a pure parental fragment is released from the recombinant molecule. Finally, when $\mathrm{CM}$ is added between 7 and $9 \mathrm{~min}$ after infection, the parental fragment becomes covalently bound to the adjacent progeny strand and is not released by denaturation. The resulting recombinant molecule thus resembles recombinant molecules isolated from the progeny phage in which, as previously proven, the continuity of the polynucleotide chain is repaired after recombination $(9,15)$. The covalent repair of the polynucleotide chain continuity after breakage and rejoining was subsequently demonstrated for interparental recombination in T4 phage $(11,16 a)$ and also suggested for a recombinant molecule of $\lambda$ phage (14a).

\section{MAterials AND MethodS}

The materials and methods used in this paper were similar to those described in previous papers (9; Kozinski and Lorkiewicz, Proc. Natl. Acad. Sci, in press). Special meanings of terms used are given below.

Heavy and light. These terms are used to indicate that a compound is (heavy) or is not (light) substituted with 5-bromodeoxyuridine.

Phage equivalent length or unit. If referring to sedimentation velocity, phage equivalent unit implies a group of molecules sedimenting at the same rate as an intact reference molecule, which is assumed to be linear. If referring to electron microscopy, it implies a molecule of a length equal to that of mature phage DNA. If referring to denatured DNA, one phage equivalent corresponds to one intact strand.

Round of replication. A round of replication is the amount of synthesis of new DNA that is equivalent to the input DNA, e.g., complete replication of the parental molecule resulting in the production of two progeny molecules.

Replicative hybrid. A replicative hybrid is a molecule resulting from one round of replication in a medium composed of precursors of a different density label than that used for the preparation of the parental phage.

Hybrid. A hybrid is composed of one parental strand (usually radioactive) and one progeny strand (usually nonradioactive), but of a different density. Because of the linearity of the $\mathrm{CsCl}$ gradient, in a $\mathrm{CsCl}$ fractionation hybrid bands half way between the peaks of pure light and pure heavy material. For most of the data presented in this paper, the location of hybrid is calculated from a reference run in which a mixture of light ${ }^{3} \mathrm{H}$ - and heavy ${ }^{32} \mathrm{P}$-labeled DNA was banded. During the experiment, usually only light ${ }^{3} \mathrm{H}$ reference was used, but the location of the hybrid was estimated by extrapolation, with an error of less than $5 \%$.

Recombinant molecule. In the context of this paper, a molecule containing contributions from two or more molecules of different densities is called a recombinant molecule. This is not necessarily (and in the case of single infection where at a given time all progeny molecules are necessarily copies of one molecule, certainly is not) the same as a genetic recombinant.

Escherichia coli B and the osmotic shock-resistant bacteriophage mutant $\mathrm{T}_{4} \mathrm{BO}_{1}{ }^{\mathrm{r}}$ were used in all experiments. TCG medium, either light or heavy, was used for the studies of replication of DNA.

Extraction leading to the isolation of replicative DNA was performed according to the sodium dodecyl sulfate (SDS)-pronase-phenol technique described previously (12). This method was developed on the basis of a personal communication from Berns on the effect of pronase on the extraction of bacterial DNA; an 
approach which eventually was described (1a) for extraction of high-molecular-weight bacterial DNA. Denaturation was accomplished by adding alkali ( $\mathrm{KOH}$ or $\mathrm{NH}_{4} \mathrm{OH}$ ) to a final concentration of $0.2 \mathrm{M}$ and incubating for $20 \mathrm{~min}$ at $37 \mathrm{C}$.

$\mathrm{CsCl}$ fractionations were performed by adding 0.3 $\mathrm{ml}$ of DNA sample to $3.0 \mathrm{ml}$. of 9.3 molal $\mathrm{CsCl}$ prepared in citrate-salt buffer $(0.15 \mathrm{M} \mathrm{NaCl}$ plus $0.015 \mathrm{M}$

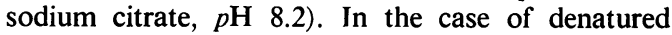
DNA, the $\mathrm{CsCl}$ was prepared in citrate-salt buffer plus $1 \%$ formaldehyde. $\mathrm{CsCl}$ centrifugations were performed at $31,000 \mathrm{rev} / \mathrm{min}$ for $72 \mathrm{hr}$ at $15 \mathrm{C}$ in a Spinco SW 39 rotor. After conclusion of the centrifugation, fractions were collected, either directly on glass-fiber filters which had been placed in scintillation vials, or, if further isolations of replicative moieties were required, in shell vials, from which $10-\mu$ liter samples were transferred to fiber-glass filters. The distribution of both isotopes was measured in a liquid scintillation spectrometer. To estimate recovery, a fraction of the $\mathrm{CsCl}$ mixture (input) was transferred, prior to centrifugation, to a fiber-glass filter, and later the counts of the fractions were summed and compared with that of the input.

Sucrose gradient fractionations were performed by overlaying 0.1 or $0.2 \mathrm{ml}$ of sample, diluted to a final concentration of less than $1 \mu \mathrm{g}$ of DNA per tube, on a 5 to $20 \%$ sucrose gradient prepared in $1.0 \mathrm{M} \mathrm{NaCl}$. In the case of the alkaline gradients, the solvent used for the preparation of the gradient was $1.0 \mathrm{M} \mathrm{NaCl}$ in $0.2 \mathrm{M} \mathrm{KOH}$. Sucrose centrifugations were at 27,000 $\mathrm{rev} / \mathrm{min}$ for $3 \mathrm{hr}$ at $15 \mathrm{C}$. After conclusion of the run, samples were collected directly on fiber-glass filters. Recovery was calculated by comparison of the input with the sum of the counts recovered in the fractions.

Occasionally the integrity of the injected strands was analyzed without previous purification of the DNA in $\mathrm{CsCl}$. In these cases, the suspensions of infected bacteria in $0.015 \mathrm{M}$ ethylenediaminetetraacetate (EDTA)- $0.15 \mathrm{M} \mathrm{NaCl}$ were supplemented with ${ }^{3} \mathrm{H}$-labeled reference phage and lysed in a final concentration of $0.2 \mathrm{M} \mathrm{KOH}$ or $0.2 \mathrm{M} \mathrm{NH} \mathrm{NH}_{4} \mathrm{OH} 37 \mathrm{C}$ for $20 \mathrm{~min}$. This treatment gives $100 \%$ recovery of injected DNA. Its advantage is that it bypasses phenol extraction of both intracellular and reference DNA and minimizes pipetting.

When necessary, the removal of $\mathrm{CsCl}$ or alkali was accomplished in a negative-pressure microdialyzer.

Relative molecular weight of DNA was calculated according to the formula of Burgi and Hershey (2); we have confirmed the applicability of their analysis to centrifugations performed with denatured DNA in alkaline sucrose gradients. (unpublished data). Methylated albumin column (MAK) fractionations were performed according to Mandel and Hershey (14), and the retention of DNA on nitrocellulose filters was determined by the method of Gillespie and Spiegelman (6).

\section{RESULTS}

Preparation of conservative, hybrid, early and late recombined parental molecules. Heavy $E$. coli $B$ were infected at a multiplicity of 3.0 with light, ${ }^{32}$ P-labeled bacteriophage. (A low multiplicity of infection of 0.1 phage per bacterium was used in numerous experiments, with similar results, although variations were due to low counts.) Samples were withdrawn at different intervals for extraction of DNA, and were subjected to the SDS-pronase-phenol procedure.

At the conclusion of a $\mathrm{CsCl}$ equilibrium run, fractions were collected in small vials, from each of which $10 \mu$ liters was transferred to a fiberglass filter for counting in a scintillation spectrometer. The rest of the material was saved for further analysis. After plotting the distribution of the isotope in the gradient, groups of fractions corresponding to the following DNA classes were pooled.

Conservative (C): a nonreplicated structure composed of parental DNA that is $100 \%$ light was isolated from bacteria at 9 min after infection. At that time, depending on the viability of the parental phage, the amount of the conservative class varied between 5 and $30 \%$ of the total parental label.

Hybrid (H): composed of equal parts of parental light, and progeny heavy material, at 7 min after infection; at that time, the hybrid class contained 40 to $80 \%$ of the total parental label. [The colinear association of strands in a hybrid and the argument that its density is caused solely by the equal proportion of light and heavy DNA was discussed in our previous papers $(8,9,12)$. The noticeable skew of replicative DNA toward the heavier side of the gradient (from 1.700 to 1.702) which can be observed at $6 \mathrm{~min}$ after infection, if no density label is used during the experiment, and if in $\mathrm{CsCl}$ fractionations a more shallow gradient is used, cannot affect the validity in the estimation of the proportional composition of hybrid. Hybrid composed of one light and one 5-bromodeoxyuridine-substituted strand has a density of 1.750 .]

Early recombined (E-R): molecules at $9 \mathrm{~min}$ after infection (composed of approximately $25 \%$ light, parental and $75 \%$ heavy, progeny material). Whereas hybrid and conservative DNA were well-defined classes, recombined classes were inhomogeneous in $\mathrm{CsCl}$. The early recombined class described in this paper contained approximately $30 \%$ of the total mass.

Late recombined (L-R): molecules at $13 \mathrm{~min}$ after infection (composed of 10 to $15 \%$ parental material and 85 to $90 \%$ progeny material); at that time, molecules in this class comprised between 50 and $90 \%$ of the total parental label.

The densities of most of these DNA classes were confirmed in the experiment represented in Fig. 1.

Sucrose and $\mathrm{CsCl}$ density gradient analysis of native and denatured replicative classes. The purpose of these estimations was to compare the sedimentation characteristics of the replicative 


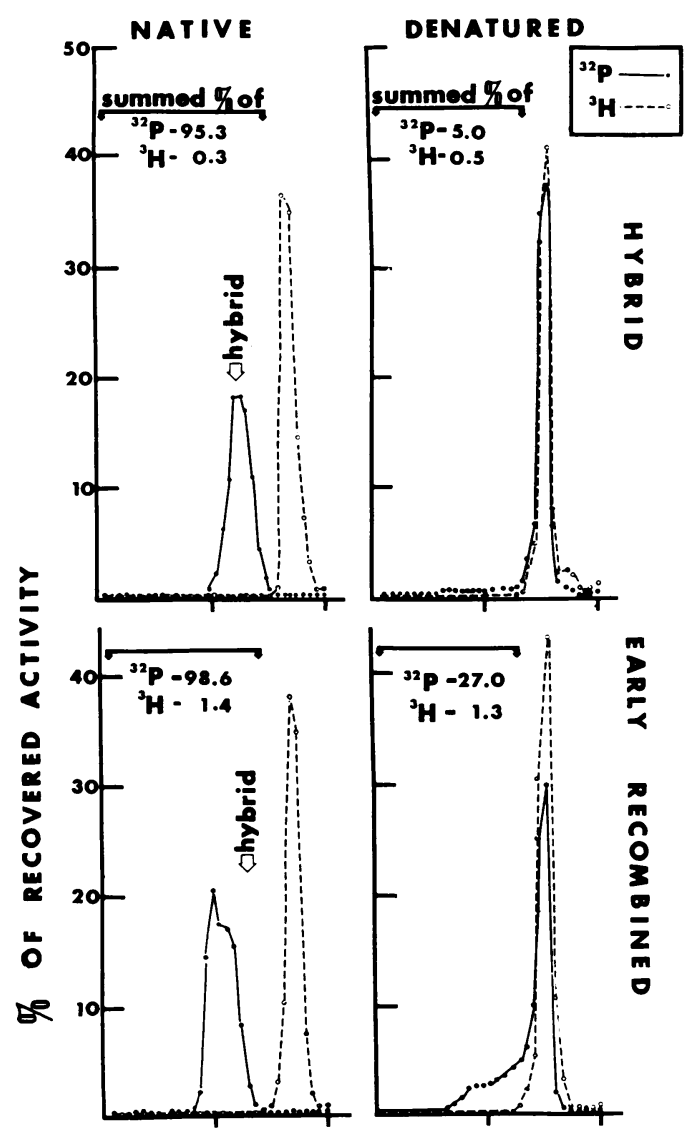

classes with ${ }^{3} \mathrm{H}$-labeled reference phage DNA. Sedimentation characteristics of the hybrid class are of special value, as will be made apparent in the Discussion. Simultaneous denaturation of replicative and reference DNA allowed us to follow, in an alkaline sucrose gradient, the integrity of the parental labeled DNA throughout the different stages of replication.

The fractions described above were dialyzed against citrate-salt buffer and supplemented with freshly prepared ${ }^{3} \mathrm{H}$-labeled (whole molecules) reference DNA. The solution was appropriately diluted and overlaid on a sucrose gradient, under normal and denatured conditions. (For a full description, see Fig. 2.) It is clear in Fig. 2 that the efficiency of recovery for conservative DNA was very good, whereas the replicated fractions were mostly sedimented to the bottom of the tube. [Sedimentation velocity centrifugations were also performed at much slower speeds, at which the reference DNA (molecular size 1) banded close to the top of the tube, and the ${ }^{32} \mathrm{P}$ formed an inhomogeneous peak. The relative molecular weight could not be calculated with any precision from this type of run, as under these conditions measurement of the distance separating reference DNA from the top of the tube is liable to significant error. However, two conclusions may be drawn from these fractionations: first, that all of the ${ }^{32} \mathrm{P}$ was recovered within the fractions, and, second, that the labeled hybrid DNA banded as a very inhomogeneous peak, in the area of the gradient where molecules of the sizes of 2 to 16 phage equivalents should be recovered.]

To emphasize further the difference between the reference and replicative DNA, the following analytical values were calculated throughout. First, the efficiency of recovery of the parental label was estimated according to the procedure described in Fig. 2, and the results are expressed as " $E$ " in the upper right corner of each panel. Second, the amount of recovery in the fractions encompassed between the two solid arrows was summed and is expressed in the figures below the solid line in the left corner. Those figures represent the total amount of either isotope in

of ice-cold EDTA, sedimented, resuspended in $0.5 \mathrm{ml}$ of EDT $A$, and lysed at a final concentration of $1 \% S D S$. DNA was extracted by the SDS-pronase-phenol method (12). Fractions were collected from the CsCl centrifugation in shell vials, as described in Materials and Methods. Samples corresponding to conservative, hybrid, earlyrecombined, and late-recombined molecules (see text) were pooled and dialyzed against citrate-salt buffer. Reruns of the first three of these, to which light, ${ }^{3} \mathrm{H}$ labeled DNA (density, $1.700 \mathrm{~g} / \mathrm{ml}$ ) was added as a reference, are presented in the figure. The ordinates are percentage of total recovery per fraction.

FIG. 1. CsCl density equilibrium analysis of native and denatured replicative moieties. Escherichia coli $B 23$ at a concentration of $3 \times 10^{8}$ in normal TCG were diluted 1:4 into heavy TCG and grown for two generations. These bacteria were then infected with light, ${ }^{32} P$-labeled $T 4$ phage. The multiplicity of infection was 3.0. Samples $(5.0 \mathrm{ml})$ were withdrawn at $6,7,9$, and 13 min after infection; the samples were diluted with $5.0 \mathrm{ml}$ 


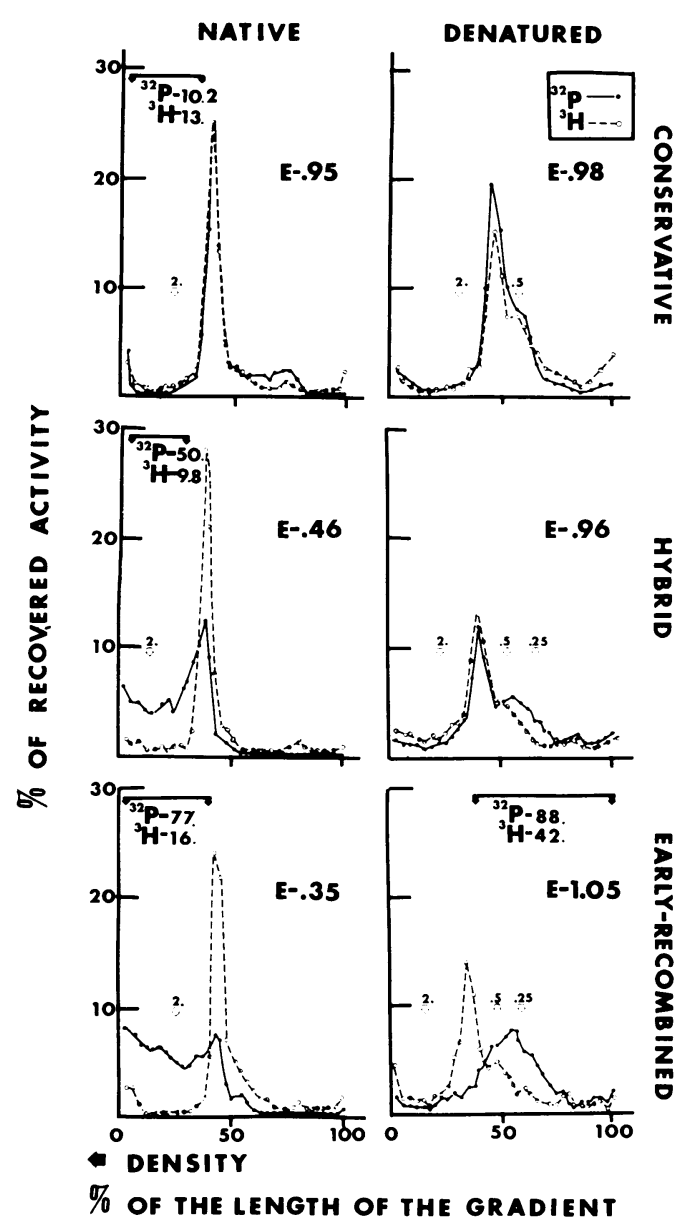

Fig. 2. Sucrose gradient analysis of replicative moieties isolated from CsCl. Samples of the DNA prepared as described in Fig. 1 were analyzed in sucrose gradients. The experimental details are presented in Materials and Methods. The DNA was diluted to less than $10 \mu \mathrm{g} / \mathrm{ml}$, and between $3 \times 10^{4}$ and $1 \times 10^{5}$ counts per min per $\mathrm{ml}$, and $0.1 \mathrm{ml}$ was used for each gradient. The efficiency of recovery, indicated in each figure with $E$, was computed as

$$
E=\frac{{ }^{3} H /{ }^{32} P(\text { Input })}{{ }^{3} H /{ }^{32} P(\text { Recovered })}
$$

and measures the relative recovery of ${ }^{32} P$ with respect to the reference ${ }^{3} \mathrm{H}$. The figures between the solid black arrows show the total percentage recovery in the region indicated. The open arrows represent the locations expected of molecules of the sizes indicated, relative to the ${ }^{3} H$-reference DNA considered as size 1.

the region of the gradient where molecules larger than one phage equivalent unit should be located. For instance, for hybrid DNA, 54\% (unrecovered) of the input DNA settles to the bottom of the tube, behaving as if it were incorporated into molecules larger than four phage equivalent units, whereas $50 \%$ of the recovered activity ( $25 \%$ of the total input) forms a heterogeneous population of molecules sedimenting more quickly than the reference molecule of size 1 .

In the case of denatured DNA (lower right panel), the total fraction of recovered isotopes was summed in the area between the reference label and the top of the tube. This was done to emphasize the amount of ${ }^{32} \mathrm{P}$-labeled DNA that is smaller than one phage equivalent unit.

It is obvious that, upon denaturation, both conservative and hybrid DNA release singlestranded subunits similar in size to the denatured reference DNA. The recombined DNA, however, releases a considerable amount of ${ }^{32} \mathrm{P}$ in pieces smaller than the reference molecules when denatured, indicating that there is a large number of single-stranded breaks.

The sucrose gradient analysis of the replicative moiety was performed after a $\mathrm{CsCl}$ fractionation, and, moreover, in parallel with reanalysis in $\mathrm{CsCl}$ (Fig. 1). We thus eliminated the possibility that the "large molecule," being fragile, does not survive the treatment of fractionation. During extraction, we may introduce artificial breaks, but whatever survives these breaks still displays a very large size.

The results of the $\mathrm{CsCl}$ analysis of native and denatured DNA are presented in Fig. 1. The conclusion from Fig. 1 is clearly that, after denaturation, the hybrid DNA releases pure parentlike fragments. The early recombined DNA releases some fragments containing both progeny and parental DNA, and in the late recombined DNA almost all of the parental DNA has become covalently bonded to the progeny DNA. These results for hybrid and early recombined DNA, together with the analysis shown in Fig. 2, are inconsistent with the terminal additions, by covalent bonding, of a new strand to the old. They also indicate that the early recombined molecules contain short parental fragments with a singlestranded break at each end, whereas in the late recombinant molecules (Fig. 1) the parental fragments become covalently linked with the newly synthesized heavy DNA and the breaks are repaired.

Electron microscopy of hybrid DNA. The unusually fast sedimentation of hybrid molecules described above led to the assumption that the sedimentation might be due to a "more compact" structure rather than to enormous lengths. Electron microscopy of conservative, hybrid, and reference DNA was performed for two reasons. Of primary importance was the search for possible differences in the conformation pattern of hybrid; this included such anomalies as loop formation, puffing, circularization or compact- 


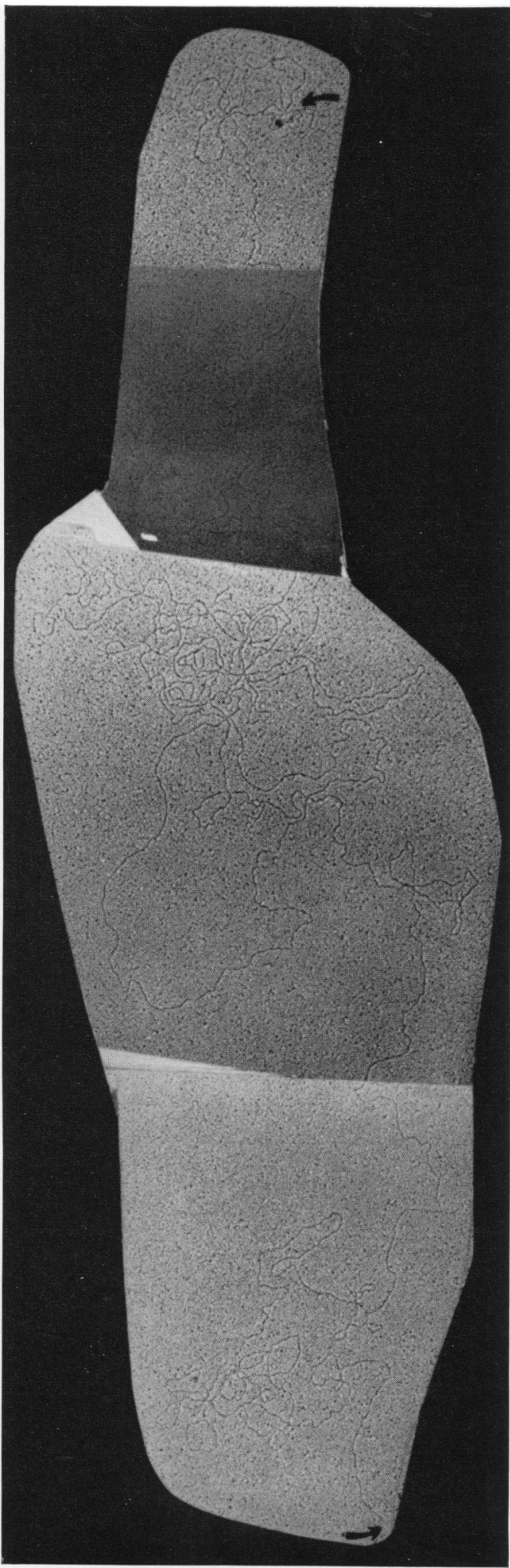

ness of structure. Secondly, electron micrographs also enabled us to measure the length of the molecules.

The sedimentation and density characteristics of the DNA used in these studies are represented in Fig. 1 and 2. DNA was prepared for electron microscopy according to the Kleinschmidt technique (7). Representative micrographs are shown in Fig. 3 and 4.

Although conservative DNA (Fig. 3) is indistinguishable from reference DNA, molecules of hybrid (Fig. 4) are highly tangled structures covering a considerably smaller area per unit of length (A). The replicative molecules also display numerous branchings, loops and puffs, possibly formed by single-stranded or partially singlestranded areas. $B_{1}$ in Fig. 4 illustrates this point, whereas $B_{2}$ and $B_{3}$ more than likely represent double-stranded puffs and loops.

The extent of tangling is not dependent on circularization, since even short fragments tend to form highly compact and twisted structures. In the stretched-out segments of the replicative molecules, numerous kinks are visible, as indicated by the arrows on Fig. 4C.

In summary, brief inspection of the micrographs in Fig. 4, which are quite representative for the appearance of hybrid molecules in electron microscope preparations, allows us to conclude that, indeed, hybrid DNA is considerably different from conservative DNA. Compared with the hybrid DNA, conservative molecules occupy a large area and, as is easily noticeable in Fig. 3, have to be traced and reconstituted from several electron micrographs. An additional feature observed in the replicative DNA is that, in approximately $30 \%$ of the molecules of the size equal to one phage equivalent $(55 \mu)$ or larger, no ends were apparent. However, the true number of circular molecules might be larger, since in some structures a relatively long kink might be mistakenly considered as the end of the molecule.

An attempt to estimate the length of the repli-

FIG. 3. Typical nonreplicated conservative molecule isolated from $\mathrm{CsCl}$. The isolated conservative $D N A$, prepared as described in Fig. 1, was mixed with cytochrome $c$ and spread on $0.3 \quad$ i $\mathrm{NH}_{4}$ acetate buffer (a useful personal suggestion from Freifolder). The grids were streaked across the monolayer film, dried, and shadowed with platinum. The molecule has a total length of $57 \mu$ and represents a typical nonreplicated molecule. It is indistinguishatle from the control, mature phage molecules, not shown in this paper. It occupies a large area, as can be seen from the picture, and was reconstituted from several electron micrographs. For the sake of consistency the length of this molecule was measured in the same manner which was described for Fig. 4. 


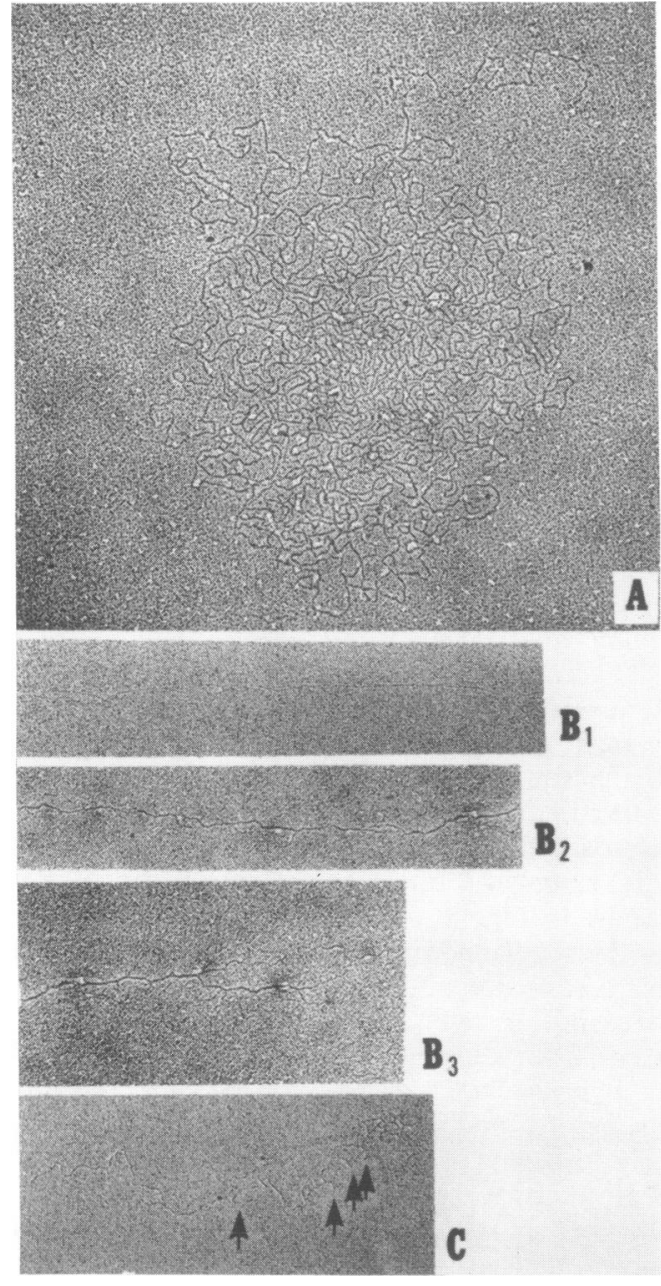

Fig. 4. Typical replicated molecule isolated from a hybrid moiety in $\mathrm{CsCl}$. The isolated hybrid DNA was mixed with cytochrome $c$ and spread on a $0.3 \mathrm{M} \mathrm{NH}_{4}$ acetate buffer. The grids were streaked across the monolayer film, dried, and shadowed with platinum. Molecule $A$ has a length of $105 \mu . B_{1}$ illustrates "puffed," most probably single-stranded areas. $B_{2}$ and $B_{3}$ illustrate numerous open areas, most likely doublestranded areas. $C$ (see arrows) represents abundant kinks found, possibly due to the presence of singlestranded areas (Kleinschmidt, personal communication). Since measuring the length of such molecules is difficult with a map measurer, the following technique was used. The molecules were traced on onion skin paper with a pen delivering a constant amount of ink per unit of length. ${ }^{32} P$ was added to the ink. The paper was sprayed with $0.1 \mathrm{M} \mathrm{Na}_{2} \mathrm{HPO}_{4}$ and dried. The tracing was then ashed and counted in the scintillation spectrometer. The length was calculated by comparison with the counts from a line of known length delivered with the same ink and pen. cative molecules can, of course, be criticized as being subject to error because of tangling, possible formation of a four-stranded structure, or possible aggregation. In our attempt to produce some approximate statistics regarding the weighted size distribution, 122 molecular "individuals" were photographed. Their lengths were estimated and summed. The molecules were then divided into four classes. (The molecules belonging to the class of large-size molecules are defined by the absence of any molecular individuals between 60 to $90 \mu$ and 30 to $45 \mu$. The division between the class considered as size $1 / 2$, and the so-called short fragments is arbitrary.)

Class 1: molecules that measured between 90 and $130 \mu$ were considered as size 2 . This class accounted for $30 \%$ of the summed length, or total mass, of all of the molecules.

Class 2: molecules that measured between 45 and $60 \mu$ were considered as size 1 . This class constituted approximately $30 \%$ of the total mass of DNA.

Class 3: molecules that measured between 15 and $30 \mu$ were considered as size $1 / 2$. This class also constituted approximately $30 \%$ of the total mass of DNA.

Class" 4: molecules shorter than $15 \mu$ were considered as a class of "short fragments," which, although numerous, constituted only $10 \%$ of the total mass of DNA.

TABLE 1. Retention of various replicative moieties on $M A K$ and nitrocellulose filters

\begin{tabular}{|c|c|c|}
\hline Type of replicative DNA & $\begin{array}{c}\text { MAK } \\
\text { fractionation }\end{array}$ & $\begin{array}{l}\text { Retention on } \\
\text { nitrocellulose }^{a}\end{array}$ \\
\hline $\begin{array}{l}\text { Conservative, } 9 \mathrm{~min} \\
\text { after infection }\end{array}$ & $E=1.2$ & $E=1.1$ \\
\hline $\begin{array}{l}\text { Conservative, } 13 \mathrm{~min} \\
\text { after infection }\end{array}$ & $E=1.1$ & $E=0.9$ \\
\hline Hybrid & $E=0.26$ & $E=2.1$ \\
\hline Early recombined & $E=0.32$ & $E=1.9$ \\
\hline Late recombined & - & $E=2.2$ \\
\hline
\end{tabular}

${ }^{a}$ These figures are reduced from the sums of counts on six filters.

$$
E=\frac{{ }^{3} \mathrm{H} /{ }^{32} \mathrm{P} \quad \text { (input) }}{{ }^{3} \mathrm{H} /{ }^{32} \mathrm{P} \text { (recovered) }}
$$

Recovered in the case of MAK filters refers to the sum of radioactivity within the fractions; therefore, values less than 1 indicate preferential retention of ${ }^{32} \mathbf{P}$. Recovered in the case of nitrocellulose retention refers to radioactivity adsorbed to the filter; therefore, values greater than 1 indicate preferential retention of ${ }^{32} \mathrm{P}$. 


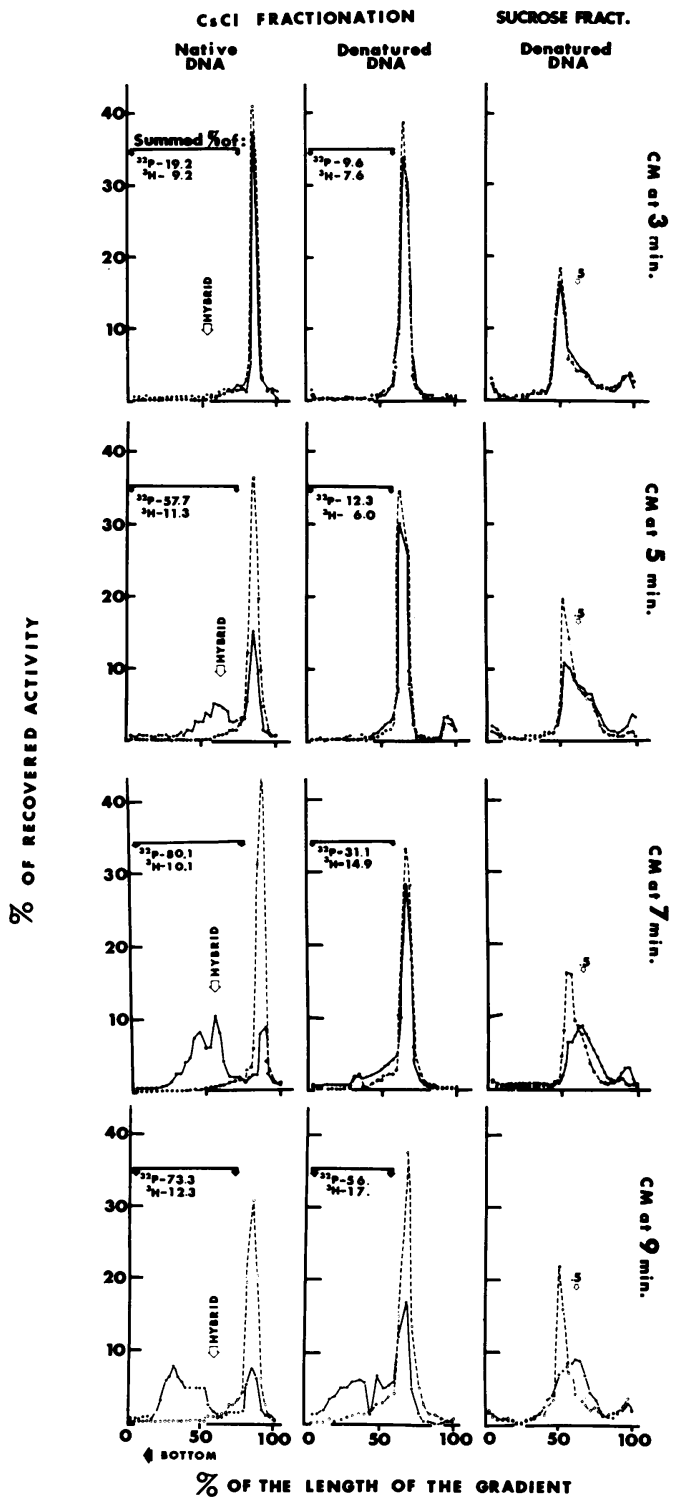

FIG. 5. CsCl and sucrose gradient analysis of intracellular DNA isolated at $45 \mathrm{~min}$ after infection from heavy bacteria infected with light, radioactive ${ }^{32} P$ bacteriophage. Escherichia coli B23 cells were grown in heavy TCG for two generations to a concentration of $3 \times 10^{8}$ bacteria $/ \mathrm{ml}$ and infected with light, ${ }^{32} \mathrm{P}$-labeled (specific activity, $3 \mathrm{mc}$ of ${ }^{32} \mathrm{P} / \mathrm{mg}$ of $P$ ) $T_{4}$ phage. The multiplicity of infection was 3 phage per bacterium. Chloramphenicol (CM) was added to portions of this

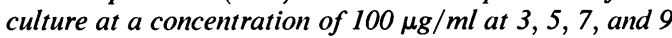
min after infection. All samples were incubated until 45 min afler infection, when DNA was extracted as described in Fig. 1. ${ }^{3} \mathrm{H}$-labeled, light reference DNA was added, and samples were analyzed in $\mathrm{CsCl}$ (native $D N A$ and denatured DNA). (Light, ${ }^{3} \mathrm{H}$-labeled phage
These statistics clearly indicate that no significant number of molecules larger than the maximum expected for hybrid could be detected in an electron microscope.

Preferential retention of replicative DNA on nitrocellulose filters and methylated albumin column $(M A K)$. The electron micrographs supported our previous conclusion (13), also indicated by the elegant enzymatic data of Frankel (4), that replicative DNA contains single-stranded regions. Such DNA should be preferentially retained on nitrocellulose filters or on a MAK column.

Conservative and replicative DNA were supplemented with ${ }^{3} \mathrm{H}$-labeled reference DNA and tested for retention on nitrocellulose filters (6) and a MAK column (14). The results are presented in Table 1 . It is clear from the data that all of the replicative DNA preparations are preferentially retained on both materials, whereas the conservative, nonreplicative fractions behave in a manner similar to the reference DNA.

The possibility of the retention of parental DNA on a nitrocellulose filter due to an association of parental DNA with ribonucleic acid (RNA) or protein seems very unlikely. The DNA is purified by phenol extraction, and then separated in a $\mathrm{CsCl}$ density gradient, which is likely to denature and remove most proteins which escaped phenol extraction from the DNA molecule. Experiments performed without the application of density labels, moreover, show that the density parental label remains virtually unaltered. Any significant association with RNA should make it heavier in a $\mathrm{CsCl}$ density gradient; alternatively, any association with protein should make it lighter.

Effect of chloramphenicol (CM) on the production of single-stranded breaks and repair. The conclusion from the density gradient analyses was that, with increasing time, single-stranded breaks appear in the parental strand which is isolated within the replicative moiety. Up to $7 \mathrm{~min}$ after infection, the parental DNA molecules of hybrid density are mostly integral. However, denaturation of the first molecular recombinants appearing

were added to part of the washed bacteria, and the mixture was lysed by incubation in $0.2 \mathrm{M} \mathrm{NH}_{4} \mathrm{OH}$ for 20 min at 37 C. A 0.1-ml amount of this was overlaid on an alkaline sucrose gradient.) The density of hybrid DNA is indicated by the open arrows in the column on the left. The open arrows on the right indicate the locations expected for molecular fragments of 0.5 phage equivalents. The numbers under the solid black lines in the $\mathrm{CsCl}$ panels refer to the total percentage of label recovered between the solid arrows. See text for discussion. 


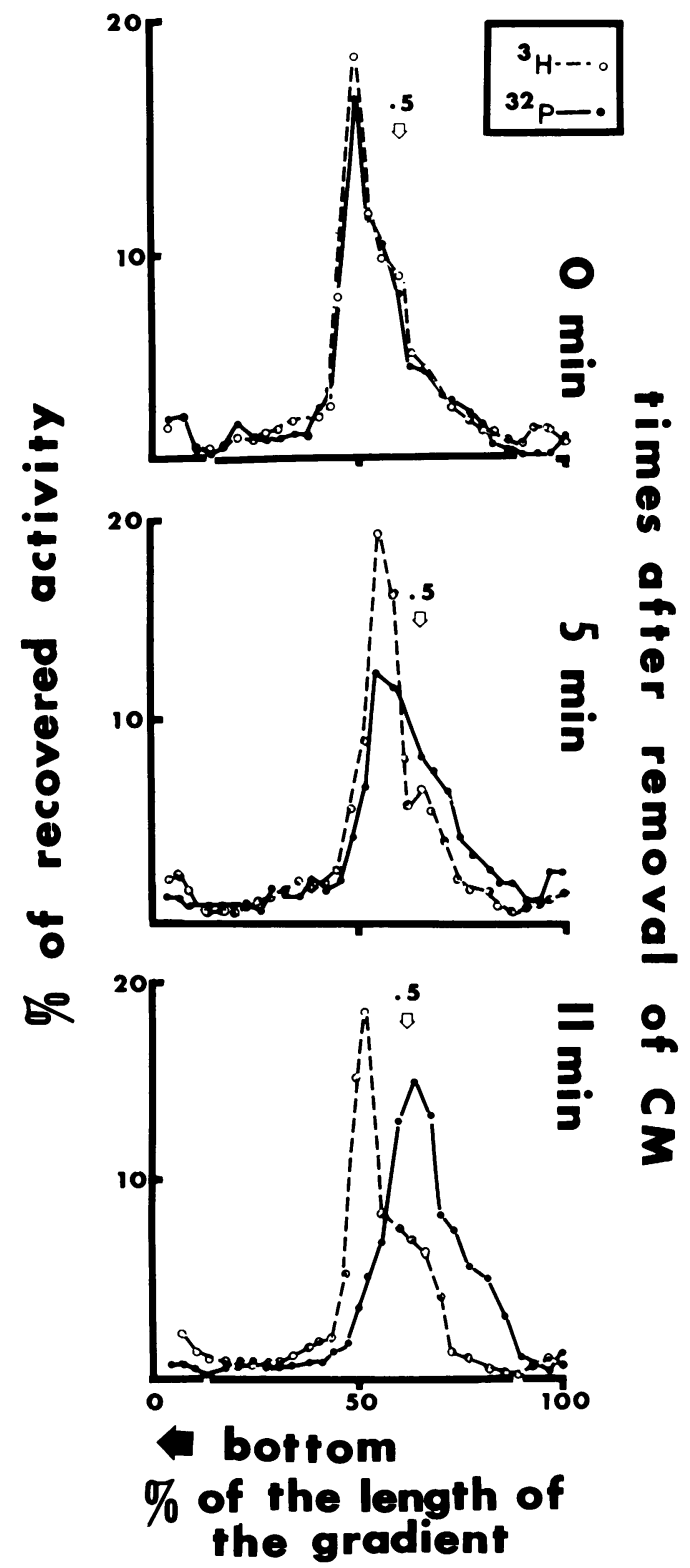

FIG. 6. Integrity of strands as a function of the time after removal of CM. Heavy, nonradioactive bacteria $\left(3 \times 10^{8}\right)$ were infected with light (multiplicity of infection, 3.0) phage. Chloramphenicol (CM), $100 \mu \mathrm{g} / \mathrm{ml}$, was added at $3 \mathrm{~min}$ after infection, and the suspension was incubated for an additional $30 \mathrm{~min}$. At this time, $C M$ was removed by filtration, and the infected bacteria were resuspended in fresh, heavy media. At intervals, samples were taken, washed with 0.015 y EDTA-0.15 M NaCl, lysed in $0.2 \mathrm{M} \mathrm{KOH}$, and analyzed in alkaline sucrose gradients. The efficiency of recovery under these conditions was $100 \%$. Note the integrity of the strands at the end of the incubation with CM and the gradual decrease after removal of $C M$. at greater than hybrid densities in $\mathrm{CsCl}$ gradients at $9 \mathrm{~min}$ after infection releases small (sucrose analyses) fragments of parental densities $(\mathrm{CsCl}$ analyses). This means that single-stranded breaks have appeared. A short time later (13 min after infection), parental strands become covalently attached to the progeny heavy strand. This suggests that break, insertion of the parental fragments, and repair of the continuity of the polynucleotide chain is not caused by a single enzymatic process. The possibility that nicking, recombination, and successive repair are catalyzed by an enzyme of host origin could not be excluded in this experimental system.

The following experiments demonstrate the inhibitory activity of $\mathrm{CM}$ on the production of nicks and recombination, and on the repair of the continuity of the polynucleotide chain, indicating that these processes are catalyzed by at least two enzymes which are more than likely coded by the bacteriophage.

Heavy bacteria were infected with light ${ }^{32} \mathrm{P}$ labeled T4 bacteriophage. CM was added at 3, 5, 7 , and $9 \mathrm{~min}$ after infection. Incubation was continued for $45 \mathrm{~min}$, at which time each of the suspensions was sedimented and resuspended in an EDTA solution. Part of each suspension was supplemented with ${ }^{3} \mathrm{H}$ light bacteriophage, lysed with $\mathrm{NH}_{4} \mathrm{OH}$, and overlaid on an alkaline sucrose gradient. DNA was extracted from the remainders of the suspensions, supplemented with ${ }^{3} \mathrm{H}$-labeled reference DNA, and analyzed both in the native and denatured forms, according to the procedure described in Fig. 5.

Enclosed between the two arrows in Fig. 5 is the total percentage of recovered activity. Note that, whereas the value of ${ }^{3} \mathrm{H}$ recovered remains constant, the mass of ${ }^{32} \mathrm{P}$ increases as a function of the time of addition of CM. The figures represented in the upper panel (CM at $3 \mathrm{~min})$ provided background for either isotope. These figures should be subtracted from the figures shown in the lower panels, and the difference should represent the net mass of replicated and recombined moieties.

When $\mathbf{C M}$ was added at 5 min after infection, $57.7 \%$ of ${ }^{32} \mathrm{P}$ assumed a density higher than that of the light reference DNA. Subtracting the background determined by the nonsynthetic system (CM at $3 \mathrm{~min}$ ), one arrives at the value of $39 \%$ native DNA which is heavier than the light reference. After denaturation, however, the total amount of DNA which was heavier than the reference ${ }^{32} \mathrm{P}$ was only $2.7 \%$ (12.3 to 9.6$)$; thus, the fraction of covalently linked DNA within the heavier-than-reference class was $2.7 / 57.7$, or roughly $5 \%$. 


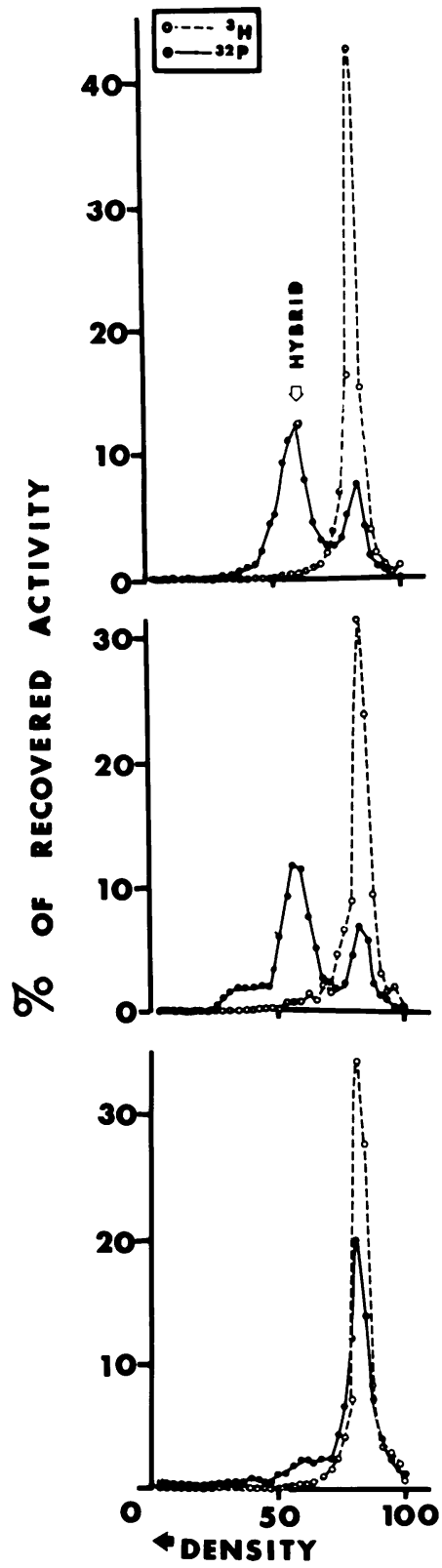

\section{\% OF the LENGTH OF the GRADIENT}

FIG. 7. Persistence of the hybrid form in chloramphenicol $(C M)$ despite concomitant synthesis of DNA and the exchange of strands in the hybrid molecule. (Net synthesis estimated in this experiment is 1.7 phage equivalent units per bacterium per min.) Heavy bacteria $\left(3 \times 10^{8} / \mathrm{ml}\right)$ were infected with light, radioactive (specific activity, $3.0 \mathrm{mc} / \mathrm{mg}$ of $P$ ) parental phage at a multiplicity of infection of 3.0. CM $(100 \mu \mathrm{g} / \mathrm{ml})$ was added at 6 min after infection. At $18 \mathrm{~min}$, one-third of
When $\mathrm{CM}$ was added at $7 \mathrm{~min}, 60.9 \%$ (corrected) of the native DNA was heavier than reference DNA. After denaturation, the corrected amount of DNA which was heavier than the reference ${ }^{32} \mathrm{P}$ was $21.5 \%$. Thus, the fraction of covalently linked DNA within the heavierthan-reference class was $35 \%$.

When CM was added at $9 \mathrm{~min}, 54.1 \%$ of the native DNA was heavier than reference DNA. After denaturation, the amount of DNA which was heavier-than-reference was $45.4 \%$, thus indicating that the fraction of covalently linked DNA within the heavier-than-reference moiety was $85 \%$.

The conclusion to be drawn from Fig. 5 is that, when CM is added at $3 \mathrm{~min}$, there is no replication, and no breaks are introduced into the parental polynucleotide chain even after 45 min of incubation. This excludes the possibility of a nicking enzyme of host origin. If CM is added at $5 \mathrm{~min}$, semiconservative replication occurs, but the parental strands in the hybrid structure are predominantly of a length equal to that of the reference material, and are almost completely separable from the heavy moiety in alkaline $\mathrm{CsCl}$. If $\mathrm{CM}$ is added at $7 \mathrm{~min}$, the size of the parental strands is smaller than the reference DNA. In this case, molecular recombination is already extensive, and the majority of the ${ }^{32} \mathrm{P}$ which is denser than the reference can be separated from the replicative complexes by denaturation. Finally, if CM is added at $9 \mathrm{~min}$, the sizes of the strands containing the parental fragments are still small, but $\mathrm{CsCl}$ analysis of the denatured recombined molecules shows that the parental pieces are for the most part united with the new, heavy DNA, presumably by covalent bonding.

In another experiment, $\mathrm{CM}$ was added at 3 min after infection, and the infected suspension

the suspension was withdrawn and sedimented in EDTA; one-third was filtered through a membrane filter, and the 5-bromodeoxyuridine medium was replaced by thymidine medium; and part remained in 5-bromodeoxyuridine medium. At $45 \mathrm{~min}$, both samples were chilled and sedimented in EDTA. DNA was extracted by the $S D S$-pronase-phenol method or by sonic treatment and was analyzed by $\mathrm{CsCl}$ density gradient centrifugation. Both yielded basically identical patterns of distribution. The figure shows results obtained with the pronase extraction. $A=18 \mathrm{~min}$ after infection. $B=45 \mathrm{~min}$ after infection. 5-Bromodeoxyuridine medium was used continuously in the presence of CM. $C=45$ min after infection. 5-Bromodeoxyuridine was placed with thymidine at $18 \mathrm{~min}$ and incubated until $45 \mathrm{~min}$ in the presence of $C M$. Note that the label returns to the parental density. 
was incubated for an additional $30 \mathrm{~min}$. $\mathrm{CM}$ was then removed by filtration through a membrane filter (Millipore Corp., Bedford, Mass.), and the bacteria were resuspended in fresh medium. At intervals, samples were taken, lysed with alkali, and analyzed in a sucrose gradient (see Fig. 6).

In agreement with our previous findings, no nicks were introduced into the parental DNA during its sojourn in the presence of CM. After removal of $\mathrm{CM}$, and with the initiation of new protein synthesis, single-stranded breaks start to accumulate.

Exchange of strands of the hybrid molecule during replication in the presence of $C M$. The previous experiment indicated that, despite continuous synthesis of DNA in the presence of CM, parental DNA reaches only a hybrid density. If one assumes that the molecule grows continuously, the hybrid stage should be transitory and would soon be replaced by a structure in which hybrid would be in the minority and could be separated from the rest of the molecule only after shearing or sonic disruption. Persistence of a hybrid-density moiety indicates the presence in the replicative pool of molecules of the size equal to one phage equivalent or maximum of size 2 . However, it was not possible to exclude the possibility that, despite concomitant synthesis of DNA, the hybrid molecule ceased participating in active replication. To test this, an experiment was performed in which the density label was reversed in the midst of the synthesis of DNA, in the presence of $\mathrm{CM}$. Under these conditions, the parental, light, ${ }^{32} \mathrm{P}$ associated with the heavy, cold strand exchanged its strand for a new, light partner, thus leaving the hybrid location and returning to the parental density in a $\mathrm{CsCl}$ analysis. Details of the experiment are presented in Fig. 7.

It is obvious that hybrid DNA actively participates in the replication process and is not merely incorporated into a continuously growing superstructure.

\section{Discussion}

The first group of experiments described in this paper is devoted to a critical re-evaluation of the size and molecular conformation of replicative T4 bacteriophage DNA. Principally, this is an extension of experiments which were published in 1965 (13). In this investigation, we were mostly concerned with partially replicated molecules (PRM) of T4 bacteriophage, i.e., molecules which had not completed one round of replication. These molecules were isolated from heavy bacteria which had been infected with light, radioactive bacteriophage. The characteristic density of these molecules in $\mathrm{CsCl}$ lay between that of light reference DNA and the hybrid density. Despite the fact that these molecules were only partially replicated, they sedimented in a sucrose gradient as if most of them were larger than two equivalents of phage DNA. We commented then that this high sedimentation rate must have been due to a change in the tertiary structure of the replicative DNA, more than likely circularization and coiling, and not due to the actual size of the molecules. We also observed that these molecules, upon denaturation, released single-stranded fragments containing the parental label, which were of the same size as reference DNA. Moreover, the density of the parental moiety of this denatured PRM was identical to that of light, denatured DNA. We thus disproved the possibility of covalent terminal additions of new strands to the parental matrix (13).

Re-evaluation of the size and conformation of replicative DNA was prompted by the recent paper of Frankel (5), who analyzed the sedimentation pattern of newly synthesized ${ }^{3} \mathrm{H}$ labled DNA and interpreted his results "as consistent with a structure for replicating T4 DNA that is considerably longer than the DNA of mature phage particles." It is obvious that, since he did not apply density labels, he could not be confronted with the logical paradox of the discrepancy arising from a several-fold increase of the apparent molecular size accompanying one (or less) round of replication. Comparison of our previous and present results with those of Frankel shows that, although our sedimentation data are within reasonable agreement, the interpretation is different.

It is obvious that the largest possible size for a hybrid molecule is the equivalent of size two, and this is only possible if one assumes that, at the conclusion of duplication, there is no separation into two progeny molecules. The replicated molecules either open to form a one-dimensional double-size molecule, or become circular. (Here, the topological possibilities might include a true circle or two interlocked circles; all of these possibilities, however, are awaiting experimental proof.) There is only one possibility for hybrid DNA to be of a size truly larger than two phage equivalents and still be composed of equal parts of old and new material; that would be the case of a recombinant arising from the union of hybrid molecules within the infected bacterium. This is contradicted by the fact that: (i) a hybrid derived from unit multiplicity of infection is equally as "large" as the hybrid derived from greater multiplicities, and (ii) there is no measurable amount of joining between the moieties contain- 
ing the parental label in bacteria infected simultaneously with light and heavy parents; this experiment was first described by Kozinski, Kozinski, and Shannon (10), and was repeated extensively by Tomizawa (1) and Kozinski and Kozinski (9). (These experiments have been performed repeatedly in our laboratory by use of a lysis and extraction procedure identical to that used in this paper. Although this is more efficient than the procedures used previously, the conclusions and essentially the results need in no way to be altered.) It seems beyond question, therefore, that a hybrid molecule of size "larger than two phage equivalents" cannot exist.

We want to emphasize the fact that, in the presence of CM, added at critical times, replication of DNA continues at a constant rate, but the parental moiety assumes only a hybrid density (10; also see Fig. 5). This hybrid, when analyzed in a sucrose gradient in the native form, displays a "very large size" identical to a hybrid isolated from a noninhibited system. The continued presence of hybrid DNA while replication occurs is inconsistent with a hypothesis of continuous growth of the molecule (visualized as continuous elongation of the double-stranded structure) because, in this case, the hybrid moiety would soon become part of a superstructure, the average density of which would be similar to that of the progeny.

The fact that parental DNA remains at a hybrid density throughout an extended period of incubation in $\mathrm{CM}$ might be interpreted as an indication that hybrid molecules are somehow unique and that, after one round of replication (resulting in the formation of hybrid), they withdraw from further replicative activities. To analyze this possibility an experiment was designed in which a light, radioactive parental phage was used to infect a heavy, nonradioactive host. CM was added at a time which would allow replication. In the midst of the synthesis, part of the suspension was separated; 5-bromodeoxyuridine was removed and replaced with thymidine. The hybrid moiety, in the case of continuous incubation in 5-bromodeoxyuridine, reached equilibrium and retained its characteristic hybrid density throughout the entire incubation period. In the bacteria where the 5-bromodeoxyuridine was replaced with thymidine, the light DNA in the hybrid moiety exchanged its partner strand for a new one, thus causing the parental label to return to the parental density. This experiment not only proves that hybrid is not a part of a supermolecule but also proves that it actively participates in replication. Neither of these facts is compatible with the hypothesis that a giant molecule grows at one end and matures on the other, as suggested in the schemes by Thomas (16) and Frankel (5). In addition, the clonal distribution of mutants and recombinants is inconsistent with this scheme, as continuous growth should not lead to the formation of a clone.

We therefore interpret the unusually fast sedimentation characteristics of replicative DNA as resulting from changes in the tertiary structure, mostly the formation of a very compact structure which can actually be observed in an electron microscope (Fig. 4). It should be emphasized that unknown cohesive forces of replicative DNA can add to aggregation in the sucrose gradient.

The possibility of the existence of nonterminal, single-stranded areas in the replicative DNA was suggested in our previous paper (13), and was more convincingly proven by Frankel (4). The data presented here on preferential retention of replicative DNA on nitrocellulose filters and on MAK, and the electron micrograph in Fig. 4B, add further evidence of single-stranded regions. Electron microscopy of the hybrid DNA reveals it as a highly tangled structure with kinks, loops, and possible single-stranded "puffs." [This point is also strengthened by the observation made by R. Miller in our laboratory that, at approximately 6 min after infection, replicative DNA becomes slightly denser than light, ${ }^{3} \mathrm{H}$-labeled reference DNA (unpublished data). The extent of this displacement suggests that $10 \%$ of the molecule is single-stranded.] Extensive twisting should not be attributed to a supercoil due to circularization, as there are numerous pictures in which even short fragments of DNA appear as highly twisted and compact structures. The estimation of the length, although liable to serious interpretational errors, indicates that hybrid molecules are smaller than, or equal to, one phage equivalent, and less frequently of the size of two molecules.

Having presented evidence against the possibility that replication occurs in a continous manner leading to the formation of very large molecules, we want to be explicit that we do not exclude the possibility that recombinational events, occurring at later times after infection, might lead to the formation of a very large structure. This large structure, however, should be a product of recombination and not replication, even though a significant amount of newly synthesized material could be incorporated into such a structure. Reduction in the size of this hypothetical structure could easily produce circularly permuted mature phage molecules. We feel that this is quite a plausible assumption.

At critical times after infection, a new enzyme 
is formed which introduces single-stranded breaks, and, at still later times, another enzyme ("repairase"), responsible for the repair of the polynucleotide chain continuity, is synthesized. [After this paper was submitted, another paper by Weiss and Richardson (17) appeared in which an enzyme "ligase" was isolated from T4-infected bacteria. It is reasonable to assume that this enzyme corresponds to "repairase" described in this paper.] This enzyme restores the integrity of the molecule after recombination.

Applying CM at different times after infection allows one to achieve a gradient of inhibition of DNA synthesizing, nicking, and repairing activities, in that order. This experiment indicates that the nicking enzyme is not of host origin, but is a phage-coded enzyme. [There is one exception to this. After injection, UV-irradiated bacteriophage T4 DNA acquires single-stranded nicks almost instantly. This nicking is not inhibited by $\mathrm{CM}$, even if it is added several minutes before infection. These nicks are obviously introduced by a host enzyme (Kozinski and Lorkiewicz, (Proc. Natl. Acad. Sci. U.S., in press).]

It is tempting to speculate that the introduction of single-stranded nicks is a prerequisite for recombination, and that the phage-coded enzyme we described as "recombinase" $(10)$ is responsible for producing these single-stranded breaks. This might explain why certain phage species, like $\lambda$, recombine very rarely, while others, like T4, recombine with very high frequency.

\section{ACKNOWLEDGMENTS}

This investigation was supported by National Science Foundation Grant GB 5283 and Public Health Service Grant 7301-Ca 10055 from the National Cancer Institute.

\section{Literature Cited}

1. Anraku, N., and J. Tomizawa. 1965. Molecular mechanisms of genetic recombination of bacteriophage. V. Two kinds of joining of parental DNA molecules. J. Mol. Biol. 12:805-815.

1a. BerNs, K. I., AND C. A. ThOMAS. 1965. Isolation of high molecular weight DNA from Hemophilus influenzae. J. Mol. Biol. 11:476-490.

2. Burgi, E., AND A. D. Hershey. 1963. Sedimentation rate as a measure of molecular weight of DNA. Biophys. J. 3:309-321.

3. Frankel, F. R. 1963. An unusual DNA extracted from bacteria infected with phage $T_{2}$. Proc. Natl. Acad. Sci. U.S. 49:366-372.

4. Frankel, F. R. 1966. The absence of mature phage DNA molecules from the replicating pool of T-even infected E. coli. J. Mol. Biol. 18:109-126.
5. Frankel, F. R. 1966. Studies on the nature of replicating DNA in $T_{4}$ infected E. coli. J. Mol. Biol. 18:127-143.

6. Gillespie, D., and S. S. Spiegleman. 1965. A quantitative assay for DNA-RNA hybrids with DNA immobilized on a membrane. J. Mol. Biol. 12:829-842.

7. Kleinschmidt, V. A., and R. K. ZahN. 1959. Uber Desoxyribonucleinsaure Molekeln ein Protein Mischfilmen. Z. Naturforsch. 14b:770779.

8. KozINSKI, A. W. 1961. Fragmentary transfer of P32-labeled parental DNA to progeny phage. Virology 13:124-134.

9. KozINSKI, A. W., AND P. B. KozINSKI. 1963. Fragmentary transfer of $\mathrm{P}^{32}$ labelled parental DNA to progeny phage. II. The average size of the transferred parental fragment. Two cycle transfer, repair of the polynucleotide chain after fragmentation. Virology 20:213-229.

10. Kozinski, A. W., P. B. Kozinski, and P. SHanNON. 1963. Replicative fragmentation in $T_{4}$ phage; inhibition by chloramphenicol. Proc. Natl. Acad. Sci. U.S. 50:746-753.

11. Kozinski, A. W., and P. B. Kozinski. 1964. Replicative fragmentation in $\mathrm{T}_{4}$ bacteriophage DNA. II. Biparental molecular recombination. Proc. Natl. Acad. Sci. U.S. 52:211-218.

12. Kozinski, A. W., AND T. H. LiN. 1965. Early intracellular events in the replication of $\mathrm{T}_{4}$ phage DNA. I. Complex formation of replicative DNA. Proc. Natl. Acad. Sci. U.S. 54:273278.

13. Kozinski, A. W., AND P. B. KozINSKI. 1965. Early intracellular events in the replication of $T_{4}$ phage DNA. II. Partially replicated DNA. Proc. Natl. Acad. Sci. U.S. 54:634-640.

14. Mandell, J., and A. D. Hershey. 1960. A fractionating column for analysis of nucleic acids. Anal. Biochem. 1:66-77.

14a. Meselson, M. 1964. On the mechanism of genetic recombination between DNA molecules. J. Mol. Biol. 9:734-745.

15. Shahn, E., and A. W. Kozinski. 1966. Fragmentary transfer of $\mathrm{P}^{32}$ labelled parental DNA to progeny phage. III. Incorporation of a single parental fragment to the progeny molecule. Virology 30:455-470.

16. Thomas, C. A. 1966. Recombination of DNA molecules. Progr. Nucleic Acid Res. 5:315-337.

16a. TomizaWA, J., AND N. ANRAKU. 1965. Molecular mechanisms of genetic recombination in bacteriophage. IV. Absence of polynucleotide interruption in DNA of T4 and $\lambda$ phage particles, with special reference to heterozygosis. J. Mol. Biol. 11 :509-527.

17. Weiss, B , aND C. C. RichaRdson. 1967. Enzymatic breakage and joining of deoxyribonucleic acid. I. Repair of single-strand breaks in DNA by an enzyme system from Escherichia coli infected with $\mathbf{T}_{4}$ bacteriophage. Proc. Natl. Acad. Sci. U.S. 57:1021-1029. 